\title{
Direct Writing of Liquids by Micro Dispensing: Stability and Shape of Laminar Jets with high Froude Numbers
}

Sreemannarayana Mypati, Aristides Docoslis, Dominik P.J. Barz ${ }^{[\operatorname{cnote}][1][1}$

Department of Chemical Engineering, Queen's University, Kingston, ON, K' 7 L $3 N 6$, Canada

\begin{abstract}
Nowadays, a wide range of industrial manufacturing processes require high precision for product manufacturing. Micro dispensing is concerned with jetting of liquids through nozzles with a typical diameter on the order of magnitude of 100 microns. In this work, various aspects of micro dispensing of a Newtonian liquid are studied, including jet break-up and the (equilibrium) profile of a printed line on a substrate. Contrary to the investigations of others, this work is concerned with jets of high Froude (Weber) numbers in the range of $40 \lesssim F r \lesssim 1500(1 \lesssim W e \lesssim 100)$. Phenomenological correlations between the liquid properties, dispensing conditions and the jet break-up ratio are inferred based on dimensional reasoning and a comprehensive set of experiments. These correlations are used to create maps that indicate stable dispensing regimes. Furthermore, when the liquid is dispensed from the nozzle, the jet diameter usually contracts over the jet length. An analytical solution of the Navier-Stokes equation, valid to describe the shape of gravity-
\end{abstract}

\footnotetext{
*Corresponding Author

URL: dominik. barz@queensu.ca (Dominik P.J. Barz )
} 
driven jets, is modified to obtain a correlation for inertia-driven jets. Finally, a correlation is derived to (numerically) obtain the three-dimensional profile of a liquid that is dispensed on a flat substrate.

Keywords: micro dispensing, jet break-up, jet shape, dimensionless analysis, phenomenological correlation

\section{Introduction}

A wide range of industrial applications, e.g. in the electronics industry, requires high precision for the manufacturing of their products [1, 2]. Material printing on substrates using direct-write micro dispensing is advantageous for prototyping, where variations in materials properties and product design are frequent [3, 4]. Unlike conventional deposition techniques, like photolithography or screen printing, direct-write micro dispensing does not require a mask and is therefore a flexible, cost effective and rapid fabrication method $[5,6,7,8]$. There are several types of direct-write micro dispensing techniques, where the material is extruded from a nozzle in form of a continuous liquid jet such as time-pressure dispensing [9], positive displacement [10] and rotary screw displacement $[11,12]$. Comprehensive review on micro dispensing techniques is available, cf. refs.[13, 14, 15].

Liquids (inks) used in micro dispensing can be distinguished as Newtonian or non-Newtonian based on their rheology. Polymer gels, nano composites and nanoparticle inks often show pseudo plastic or thixotropic NonNewtonian behavior. There are also many examples where the micro dispensing inks are Newtonian liquids, despite their complexity. These include 
nanoparticle suspensions [16, 17, 18], low filler concentration polymer composites [19] and high viscosity UV curable resins [20, 21, 22]. Newtonian inks also have important applications in other printing technologies, such as inkjet printing [23, 24]. Contrary to ink jet printing and other drop-ondemand methods, micro dispensing facilitates the processing of a wide variety of liquids with properties like high viscosity, density and surface tension [25]. Continuous dispensing of Newtonian liquids also has relevance for cooling involving the impact of liquid jets on walls [26] or bouncing liquid jets in mold casting [27]. Hence, we restrict this work to the investigation of Newtonian liquids.

For successful printing using micro dispensing, three parameters must be well defined: i) The distance between nozzle and substrate since all jets eventually break up with growing length. ii) The jet diameter when contacting the substrate, which along with the lateral nozzle velocity, determines the (initial) resolution. iii) The profile $f$ the printed material on the substrate. This is an important parameter in many applications such as electronic packaging [28], microfluidic devices [29] and micro soldering [30].

Simply, the existence of a stable vertical jet is directly related to the dispensing velocity. Figure 1a) gives a schematic relationship between velocity and the break-up ratio of a vertical jet according to Grant and Middleman [31]. The break-up ratio is defined as the length of a stable jet normalized with the diameter of the dispensing nozzle. At very low velocities, the liquid simply drips out of the nozzle and does not form a continuous jet. At higher 

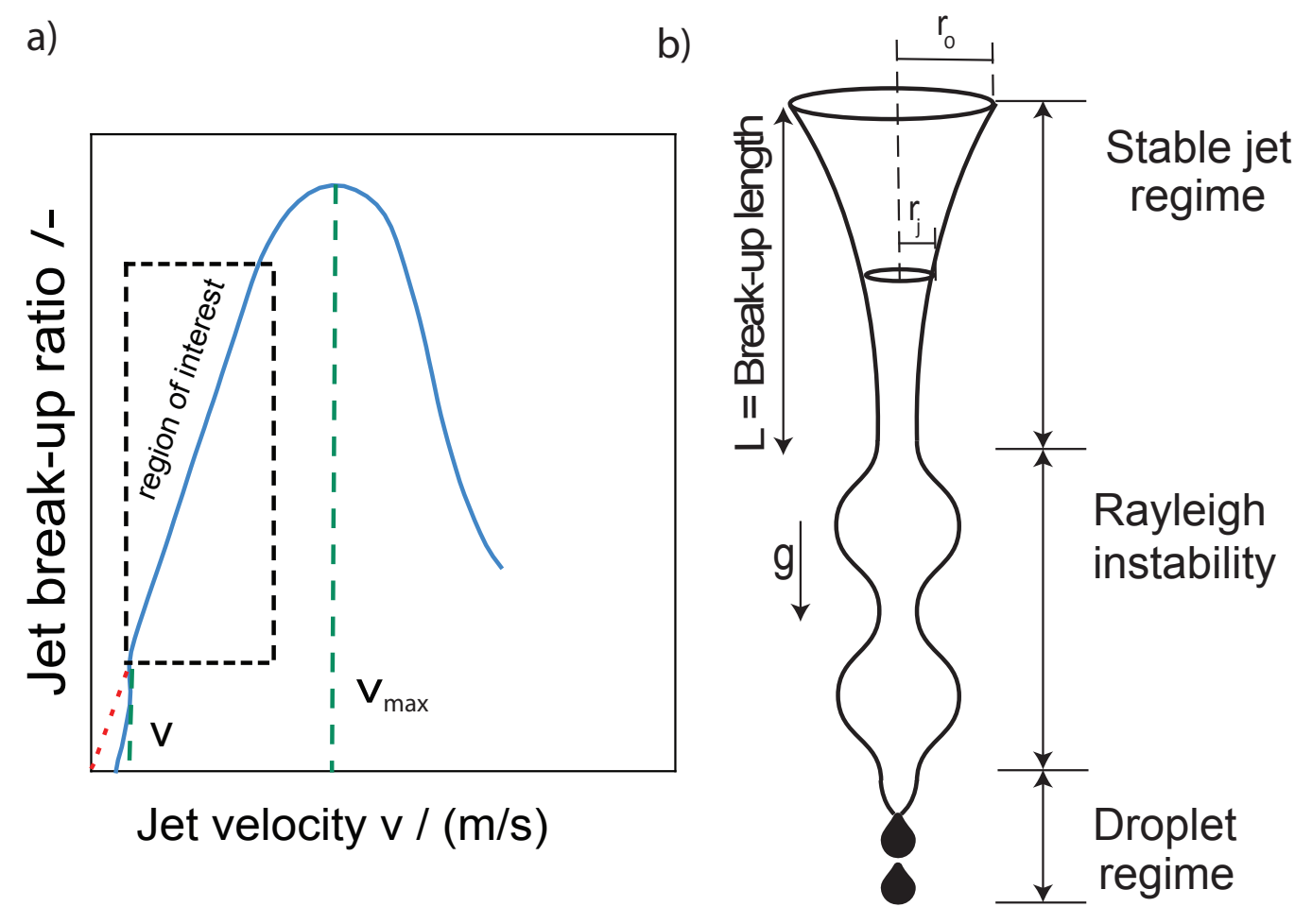

Figure 1: a) Schematic correlation between dispensing velocity of a vertical liquid jet and its break-up ratio. b) Schematic shape of the liquid jet indicating the different regimes that can occur over the jet length.

dispensing velocities, stable jets are produced and show a linear correlation between the break-up ratio and velocity. With further velocity increase, the relationship becomes nonlinear and the break-up ratio peaks and then decreases. This work focuses on the linear regime.

Figure 1b) shows the shape of a vertical cylindrical jet within the linear regime. After discharge from the nozzle, the jet is subjected to gravity. This results in a continuous constriction of the jet shape, but the interface 
remains steady; this is called the stable jet regime. After a certain length, the interface becomes rippled and then the jet breaks up into liquid drops. The jet break-up is a result of an (Rayleigh) instability that develops at the nozzle [32]. In the linear regime, it is governed by inertia along with capillary and hydrodynamic interactions at the interface [31]. Lord Rayleigh derived a relationship for the growth rate of disturbances that form on an inviscid jet surface in a vacuum as a function of the dimensionless wave number [32, 33]. This theory only considers capillarity and a linear stability analysis predicts jet break-up when the perturbation amplitude is equal to the jet radius. In practice, the break-up length also depends on gravity, surface tension and viscosity of the liquid, density difference of liquid and surrounding medium, nozzle geometry, along with other parameters [31, 34, 35, 36, 37, 38, 39]. Weber extended Rayleigh's theory by accounting for the liquid viscosity and the influence of the surrounding (non-viscous) atmosphere [34]. His correlation for the break-up length can be written in a dimensionless form as

$$
\frac{L}{d_{0}}=\ln \left(\frac{r_{m}}{\delta_{0}}\right)[1+3 O h] \sqrt{W e},
$$

where $L$ is the break-up length, $d_{0}$ is the nozzle diameter, $r_{m}$ is the (minimum) radius of the stable jet, $\delta_{0}$ is the initial disturbance factor (amplitude), and $O h$ and $W e$ are the Ohnesorge and the Weber number, respectively. The Oh number relates viscous forces to inertial and surface tension forces while the $W e$ number indicates the ratio of inertial to surface tension forces. Weber utilized the experimental data of Haenlein [40] to validate the correlation. For jets with a low velocity, he reported that $\ln \left(\frac{r_{m}}{\delta_{0}}\right)$ has an average value of 12. Later Grant and Middleman [31] slightly modified eq. (1) by introduction 
of an additional exponent. They proposed that this exponent and $\ln \left(\frac{r_{m}}{\delta_{0}}\right)$ depend on the $O h$ number as well. There are more studies concerned with jet stability; reviews can be found in refs. [41, 42].

The shape of a continuous liquid jet is the subject of numerous investigations. Generally, the diameter of the jet reduces along the jet length as a result of gravity. The actual shape of the jet interface in the linear regime is determined by gravity, velocity, density difference between jet and atmosphere, as well as viscosity of jet liquid and surrounding fluid [43, 44, 45, 46, 47, 42]. The first analytical description of jet shapes dates back to the work of Kirchhoff [48] and was based on the concept of potential flows without any external forces. Later, Scheuermann used the energy equation, considering surface tension and viscous forces, to derive a relationship between radius and length of a stable jet [49]. Several variations of Scheuermann's jet shape equation were proposed by others $[43,44,45,46]$ but the general mathematical form remains unchanged and has the dimensionless form

$$
\frac{\bar{x}}{F r}=\frac{1}{\bar{r}_{j}^{4}}+\frac{n}{\bar{r}_{j}} \frac{1}{W e}-\left(m+\frac{n}{W e}\right),
$$

where $\bar{x}=\frac{x}{r_{0}}$ and $\bar{r}_{j}=\frac{r_{j}}{r_{0}}$ are the dimensionless axial and radial position of the interface with respect to the center of the nozzle orifice, respectively; $r_{0}$ is the nozzle radius, $r_{j}$ is the jet radius at given distance $x, F r$ is the Froude number which reflects the ratio of inertial to gravitational force, and $m$ and $n$ are coefficients which are either inferred from experiments or approximative analytical solutions. Scriven and Pigford [50] used the Weisbach equation to determine the local jet radius and obtained $m=1$ and $n=0$. Kurabayashi 
[51] included the surface tension forces and proposed $m=1$ and $n=8$ (as cited in Ref.[52]). As this equation cannot be directly applied to our experiments, its modification is discussed in the results section.

Another important stage of printing is the deposition of the liquid on the solid substrate. On the one hand, there is the possibility of splashing if the jet velocity is too high and the liquid bounces back from the substrate. This is only observed for relatively long distances between the nozzle and substrate which are already critical with respect to jet stability. Therefore, splashing of liquids is not accounted for in this work. On the other hand, the dispensed liquid may spread or contract on the substrate until an equilibrium is attained; reviews concerned with (de-)wetting of simple liquids are available, cf. e.g. Refs $[53,54,55,56]$. Industrial applications generally use complex liquids that contain different components such as nano-sized particles/flakes and viscosifiers which generally change the wetting behavior [57]. Furthermore, the existing models are usually concerned with the wetting of droplets on a surface. In the case of micro dispensing, however, the liquid is printed in form of a line. Hence, this study focus on a model which connects the dispensed liquid with the equilibrium profile of a printed line on a flat surface. Wetting is considered beyond the scope of the present article.

Despite the numerous correlations that are available for jet break-up length and shape, they are usually not applicable for micro dispensing processes since the experiments were conducted in a regime where the gravitational forces are similar to the inertial forces; i.e. for $\mathrm{Fr}$ (and $W e$ ) numbers 
on the order of magnitude of one. Micro dispensing is generally done with narrow nozzles resulting in high jet velocities, and considerably larger $\mathrm{Fr}$ and $W e$ numbers.

To the best of our knowledge there are no studies which give a comprehensive treatise of (Newtonian) liquid jets under the conditions which are present for micro-dispensing. Thus, the main objectives and the novelty of the present study are: A thorough investigation of i) the break-up length and ii) the shape of the interface of a Newtonian (simple) liquid that is dispensed with high $\mathrm{Fr}$ and $W e$ numbers; and iii) the mathematical description that can be used to describe the profile of a (complex) liquid dispensed on a flat substrate. This article continues with the experimental methods and materials. Then, it proceeds with the results section, divided in break-up length, jet shape and the dispensed liquid profile, followed by concluding remarks. 


\section{Experimental Methods and Materials}

In this section, we specify the experimental setup and the materials. We conduct experiments to determine the jet break-up length, the shape of the (stable) jet before break-up and the profile of the dispensed liquid. Dispensing of the liquids is performed with a material dispenser which consists of an automated XYZ stage (Precision Valve Automation (PVA), Model DPNT0549, Cohoes, NY, USA), equipped with a micro dispensing valve FCM100. The experiments are carried out using three different dispensing nozzles having an inner diameter of $100 \mu \mathrm{m}, 250 \mu \mathrm{m}$ and $400 \mu \mathrm{m}$ along with five exemplary liquid samples. These samples are prepared such that they cover a wide range of viscosities along with a good variation in density and surface tension. To achieve this, we mix ethanol and glycerol (Reagent grade, Sigma-Aldrich Canada Company, Oakville, ON, Canada) in different ratios. The viscosity of the samples is measured using a rheometer (Model AR 2000, TA Instruments, New Castle, DE, USA) at $25{ }^{\circ} \mathrm{C}$. The surface tension is measured using the pendent drop method with a digital goniometer (Model OCA 15EC, DataPhysics Instruments GmbH, Filderstadt, Germany). The density of the sample solutions are inferred by dispensing a very defined amount of the liquid using a Hamilton micro syringe (Model Gastight 1710, Hamilton Company, Bonaduz, GR, Switzerland) and by weighing its mass with a semi-microscale balance (Model CPA225D, Sartorius, Germany). The so-measured properties of all samples, along with a commercial carbon ink that we use to determine the profile of the dispensed liquid, are summarized in Table 1. 
Table 1: Physical properties of the samples

\begin{tabular}{lllccc}
\hline Sample & $\begin{array}{l}\text { Mass } \\
\text { fraction } \\
\text { ethanol } /-\end{array}$ & $\begin{array}{c}\text { Mass } \\
\text { fraction } \\
\text { glycerol } /-\end{array}$ & $\begin{array}{c}\text { Density } \\
/ \mathrm{kg} / \mathrm{m}^{3}\end{array}$ & $\begin{array}{c}\text { Viscosity } / \\
\mathrm{kg} /(\mathrm{m} \cdot \mathrm{s})\end{array}$ & $\begin{array}{c}\text { Surface } \\
\text { tension } / \\
\mathrm{N} / \mathrm{m}\end{array}$ \\
\hline sample-1 & 0.08 & 0.92 & 1233.48 & 0.80 & 0.052 \\
sample-2 & 0.24 & 0.76 & 1178.37 & 0.27 & 0.040 \\
sample-3 & 0.48 & 0.52 & 1077.53 & 0.05 & 0.030 \\
sample-4 & 0.60 & 0.40 & 1020.27 & 0.02 & 0.025 \\
sample-5 & 0.80 & 0.20 & 916.05 & 0.005 & 0.023 \\
carbon ink & $\mathrm{N} / \mathrm{A}$ & $\mathrm{N} / \mathrm{A}$ & 1001.63 & 0.009 & 0.032 \\
\hline
\end{tabular}

The method to measure the jet break-up length is as follows: Initially, the liquids are dispensed downwards into ambient air at a constant volumetric flow rate. The flow rate is controlled by the injection pressure which can be adjusted with a micrometer located at the dispensing valve. The (average) jet velocity at the outlet is calculated from the ratio of flow rate and crosssectional area of the nozzle. A digital camera (Model DFK 23UM021, The Imaging Source, Charlotte, NC, USA) is used for capturing digital images of the jet. The images are processed with the software ImageJ (Version: 1.50i, U. S. National Institutes of Health, Bethesda, MD, USA) to evaluate the jet interface and break-up length. The experiments are performed in triplicates for each flow rate.

To evaluate the liquid profile on the substrate, we dispense a commercial carbon ink (JR-DEV 79-79-10, Novacentrix, Austin, TX, USA). As substrate, 
we utilize microscope glass slides (Fisher Scientific, Ottawa, ON, Canada). The 3D surface profile of the dispensed liquid is obtained using a surface profiler consisting of a digital microscope and a dynamic range sensor laser with a wavelength of $650 \mathrm{~nm}$ (MVP-400, DRS 500, Optical Gaging Products (OGP), Rochester NY, USA). Numerical integration of the mathematical model that describes the profile is performed with MATLAB (Version: R2018a, Mathwork, Inc, Natick, MA, USA). The MATLAB code is given in the Supporting Information to this article. 


\section{Results and Discussion}

In this section, theoretical considerations and experimental results are combined to determine the jet break-up length, shape and the profile of the dispensed liquid. In terms of break-up length, we use dimensional reasoning along with respective experiments to derive a phenomenological correlation between the dimensional groups. For the jet shape, we modify Scheuermann's equation (eq. (2)) to make it applicable for a high Froude and Weber number regime. Finally, the profile of the dispensed liquid is computed using a model that is based on the minimization of the potential energy.

\subsection{Break-up Ratio}

A proper micro dispensing process requires that the liquid is ejected towards the substrate in the form of a continuous jet. Above the critical jet length, the interface becomes unstable, cf. Figure 1b). We assume that this break-up length can be correlated to the independent quantities: nozzle diameter, jet velocity at the nozzle orifice, liquid dynamic viscosity, acceleration due to gravity, liquid density, and liquid-gas interfacial tension according to

$$
L=f\left(d_{0}, v, \mu, g, \rho, \sigma\right)
$$

Here, $f$ is a functional relationship to be derived by the rules of dimensional analysis. In contrast to other correlations from literature, we include the gravitational force in our considerations. Our jets feature high $F r$ numbers which indicate that they are governed by inertial rather than gravitational forces. Nevertheless, we assume that gravity contributes to the elongation of the jet reducing its radius and therefore influences the break-up length. 
In preparation for applying the Buckingham $\Pi$ theorem [58] to eq. (3), we note that we have $n=6$ relevant (independent) quantities and that the fundamental physical dimensions are length, mass, and time; i.e., $m=3$. According to the Buckingham $\Pi$ theorem $(n+1)-m=4$ dimensionless groups are required for the functional relationship. After some transformations, we arrive at the critical break-up ratio, Froude, Weber and Capillary number; that is

$$
N_{\text {crit }}=\frac{L}{d_{0}}, \quad F r=\frac{v^{2}}{g d_{0}}, \quad W e=\frac{v^{2} d_{0} \rho}{\sigma}, \quad C a=\frac{v \mu}{\sigma} .
$$

Despite the reduction from six to four independent quantities, there are still many experiments needed to infer a correlation. While the amount of experiments may be manageable, it is difficult to vary only one of the dimensionless groups at a time. For example, when we vary the dispensing velocity in the experiment, the $W e$ number changes along with all other (independent) dimensionless groups. Hence, we further reduce the number of dimensional groups to ease the experimental complexities. Here, we follow eq. (1) and combine the $C a$ and $W e$ number to the Ohnesorge number

$$
O h=\frac{C a}{\sqrt{W e}}=\frac{\mu}{\sqrt{\rho \sigma d_{0}}} .
$$

Hence, we arrive in the functional relationship

$$
N_{\text {crit }}=F(O h, F r)
$$

which also has the advantage that the $O h$ number can be varied by tailoring the liquid properties while the $F r$ number is independently controlled by the flow rate through the nozzle. Note that we also perform a regression for $N_{\text {crit }}=F(O h, W e)$ of our experimental data. However, the resulting 
correlation is not satisfying which justifies our approach to consider the gravitational force.

First of all, we examine whether our experiments are within the linear regime of the jet break-up. Figure 2a) shows electronic images of a dispensed liquid with $O h=0.05$ and increasing $F r$ numbers. The first image shows the situation for $F r=62$, where the kinetic energy is not sufficient to form a stable jet and the liquid drips from the nozzle. For sufficiently high $\mathrm{Fr}$ numbers, a continuous jet with a defined length can be observed. The jet elongates with increasing $F r$ numbers as can be seen by comparison of the images at $F r=148,224$ and 718. Note that the $F r$ number contains the velocity squared and therefore the correlation between $F r$ number and $N_{\text {crit }}$ is nonlinear. Hence, we plot the break-up ratio versus the jet velocity for the range that we consider in our experiments in Figure $2 \mathrm{~b}$ ). The relationship is, to good approximation, linear confirming that our experiments are conducted in the (formal) validity range of eq. (1). A respective comparison, using a constant value of $\ln \left(\frac{r_{m}}{\delta_{0}}\right)=12$, shows large discrepancies with our experiments. The differences are so significant that they cannot be mitigated by adjusting the constant which indicates that eq. (1) is not valid for a high $W e$ and $\mathrm{Fr}$ number dispensing regime.

\subsubsection{Influence of Froude and Ohnesorge Number}

To infer the influence of the $O h$ and the $F r$ number on $N_{c r i t}$, a set of experiments is performed, where we keep one of the dimensionless groups approximately constant but vary the other. Figure 3) gives the relationship between $N_{\text {crit }}$ and $\mathrm{Fr}$ for constant values of the $\mathrm{Oh}$ number. For the ease 

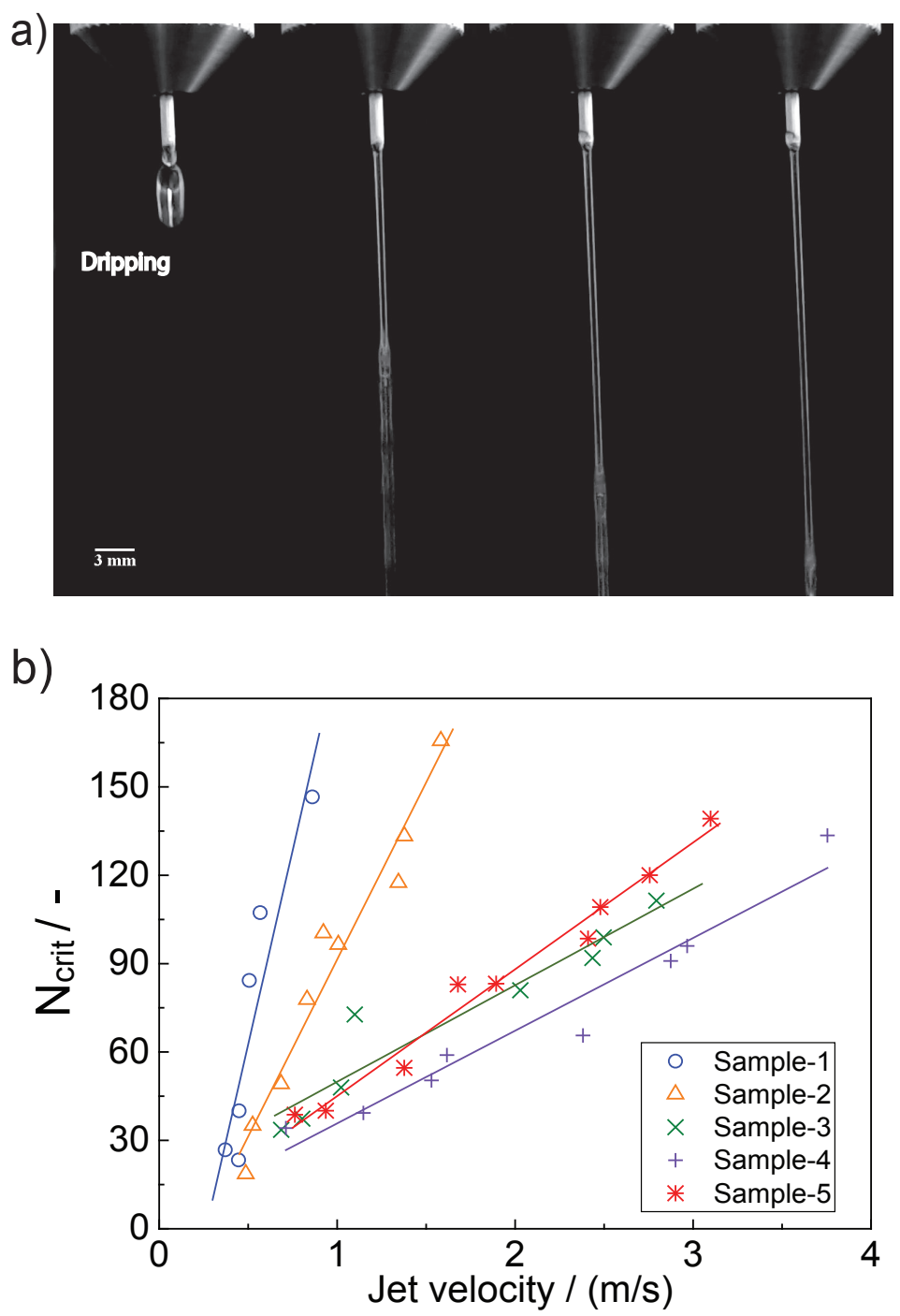

Figure 2: a) Images of jets with different $F r$ numbers but a constant $O h$ number of 0.05 . From left to right: Dripping at $F r=62$; Continuous jet with increasing length for $F r=$ 148, 224, and 718; b) Break-up ratio versus the jet velocity for different samples. 
of interpretation, we plot the logarithm of the dimensionless groups and distinguish between a low and a high $O h$ number range.

In detail, Figure 3a) gives $N_{\text {crit }}$ versus the $\mathrm{Fr}$ number for $\mathrm{Oh}$ numbers in the range from 0.07 to 2.5. For a given $O h$ number, we find that $N_{\text {crit }}$ increases when the $F r$ number increases. The correlation is, on a $\log -\log$ scale, to good approximation linear and all curves have similar slopes. Figure $3 \mathrm{~b}$ ) shows the relationship between $N_{\text {crit }}$ and $F r$ for experiments in the range of 4 to 10 . We again identify linear correlations for experiments with a constant $O h$ number. Like with the low $O h$ number range, we see that the slopes of these curves are similar but the same but the slopes are considerably steeper which confirms the division into two $O h$ number ranges. In either range, we cannot identify the influence of the $O h$ number which results in a data collapse on a single line in order to derive a scaling law. In terms of a physical interpretation, our results clarify that inertia stabilizes the jet. In other words, the higher the dispensing velocity ( $F r$ number), and the lower the influence of the gravitational force, the higher the break-up ratio of a jet.

To get further insights into the dependencies, we plot the logarithm of the break-up ratio $N_{\text {crit }}$ versus the logarithm of the $O h$ number for a given Fr number in Figure 4. Note that due to the nature of our experimental setup, it is rather difficult to keep the Fr number strictly constant. Hence, we cluster the experimental data in a way that the average $F r$ numbers between clusters have a significant difference while maintaining a relative narrow variation within a data group. Here, we have disregarded about 50 
a)

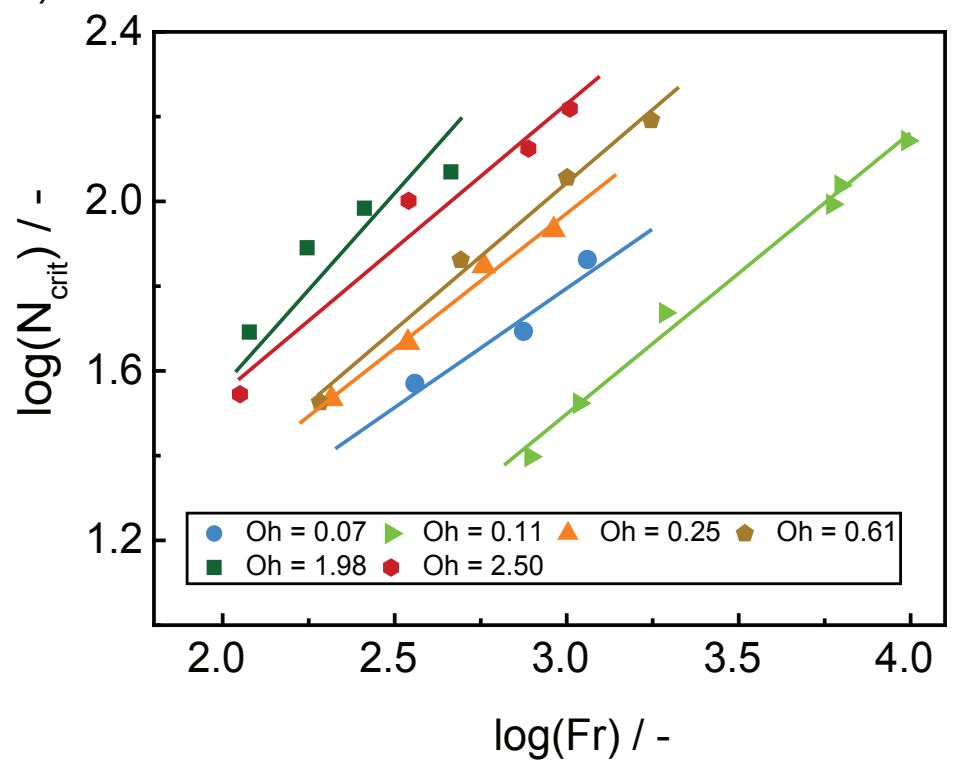

b)

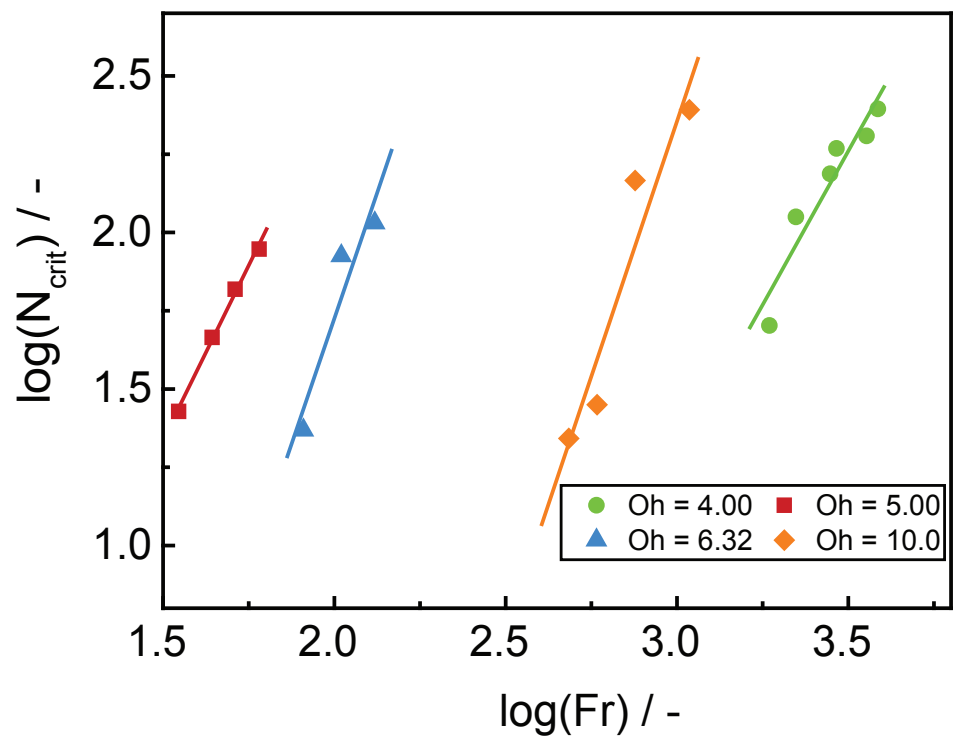

Figure 3: Break-up ratio versus the Froude number for constant values of the Ohnesorge number: a) Low range with $0.07 \leq O h \leq 2.5$ and b) High range with $4 \leq O h \leq 10$. Lines are from linear regression of data with constant Ohnesorge number. 
a)
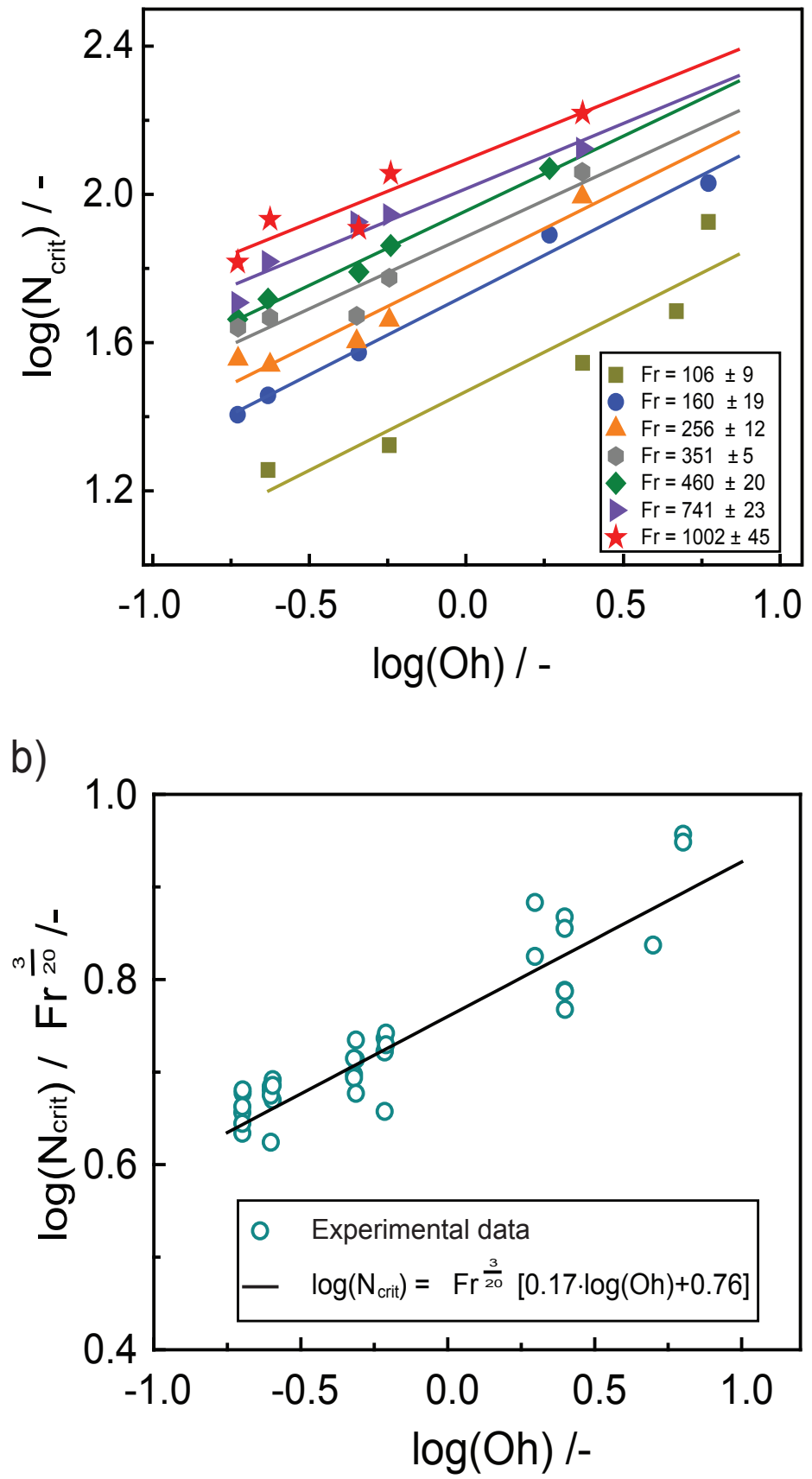

Figure 4: a) Break-up ratio and b) scaled break-up ratio versus the Ohnesorge number for constant (average) values of the Froude numbers. 
$\%$ of the experiments because they cannot be grouped and the remaining experiments are mainly in the low $\mathrm{Oh}$ range. We observe that for a given $\mathrm{Fr}$ number, the break-up ratio increases when the $O h$ number increases. The correlations between $N_{c r i t}$ and $O h$ number are linear and have similar slopes, to good approximation. It seems that the Fr number merely shifts the curves on the $y$-axis. We perform a scaling analysis and find that all data collapse on a single curve, to good agreement, when we scale (normalize) the breakup ratio with $\mathrm{Fr}^{\frac{3}{20}}$ as shown in Figure 4b). Hence, we perform a regression and infer that the data in the low $O h$ number range can be very reasonably described by

$$
\log \left(N_{c r i t}\right) \simeq \operatorname{Fr}^{\frac{3}{20}}(0.17 \cdot \log (O h)+0.76)
$$

with a coefficient of determination of 0.82 . The correlation is a good description, as demonstrated in the parity plot $4 \mathrm{~b}$ ), for jets up to $F r \simeq 1000$ and $O h \simeq 3$. In terms of physical interpretation, we recall that the $O h$ number can be considered as the ratio of viscous and inertial and surface tension forces $O h \sim F_{\text {vis }} / \sqrt{F_{\text {in }} F_{\text {cap }}}$. The results indicate that for a constant jet velocity (inertial force), increasing the viscosity and/or lowering the surface tension stabilizes the jet.

We perform two further multivariable regressions for the high $O h$ number range as well as for the entire set of experimental data. We infer the significance of single terms in the correlation by analysis of the variance and accordingly incorporate or discard them. The regression of the high range data with $4 \leq O h \leq 10$ gives

$$
N_{\text {crit }} \approx 93.6-0.07 \mathrm{Fr}-11 \mathrm{Oh}+0.03 \mathrm{Fr} \cdot \mathrm{Oh},
$$


with a coefficient of determination of 0.74 . The regression of all experimental data results in

$$
N_{\text {crit }} \approx 32.38+0.04 \mathrm{Fr}+9.43 \mathrm{Oh}+0.001 \mathrm{Fr} \cdot \mathrm{Oh}
$$

having a coefficient of determination of 0.73 . The very similar coefficients of determination reveal that the regression of the high $O h$ number range does not give a real advantage. Hence, we use correlation eq. (9) for constructing a dispensing map in the next section.

\subsubsection{Liquid Dispensing Map}

Based on our work, it is possible to construct a liquid dispensing map which indicates different jet regimes depending on liquid as well as dispensing properties. This map can be used to choose proper micro dispensing conditions of various liquids, flow rates, nozzle diameters, and spacing between nozzle and substrate. More specifically, Figure 5 comprises the liquid dispensing map which is constructed from a combination of our experimental results and the phenomenological correlation between the dimensionless groups given in eq. (9). The region spanning the entire range of $\mathrm{Fr}$ numbers and below a $O h$ number of around 0.05, features the regime where the liquids generally have a very low viscosity. Although continuous jets can be dispensed in this regime, they are hard to control, decay rapidly and usually have low break-up ratios, which makes this area rather unsuitable for micro dispensing. For Oh numbers larger than around 0.05, the map can be divided into four different regions. These regions are separated by break-up ratio isolines obtained from the regression derived in Section 3.1.1 except the isoline for $N_{\text {crit }}=0$ which is directly obtained from experimental data. Based on 


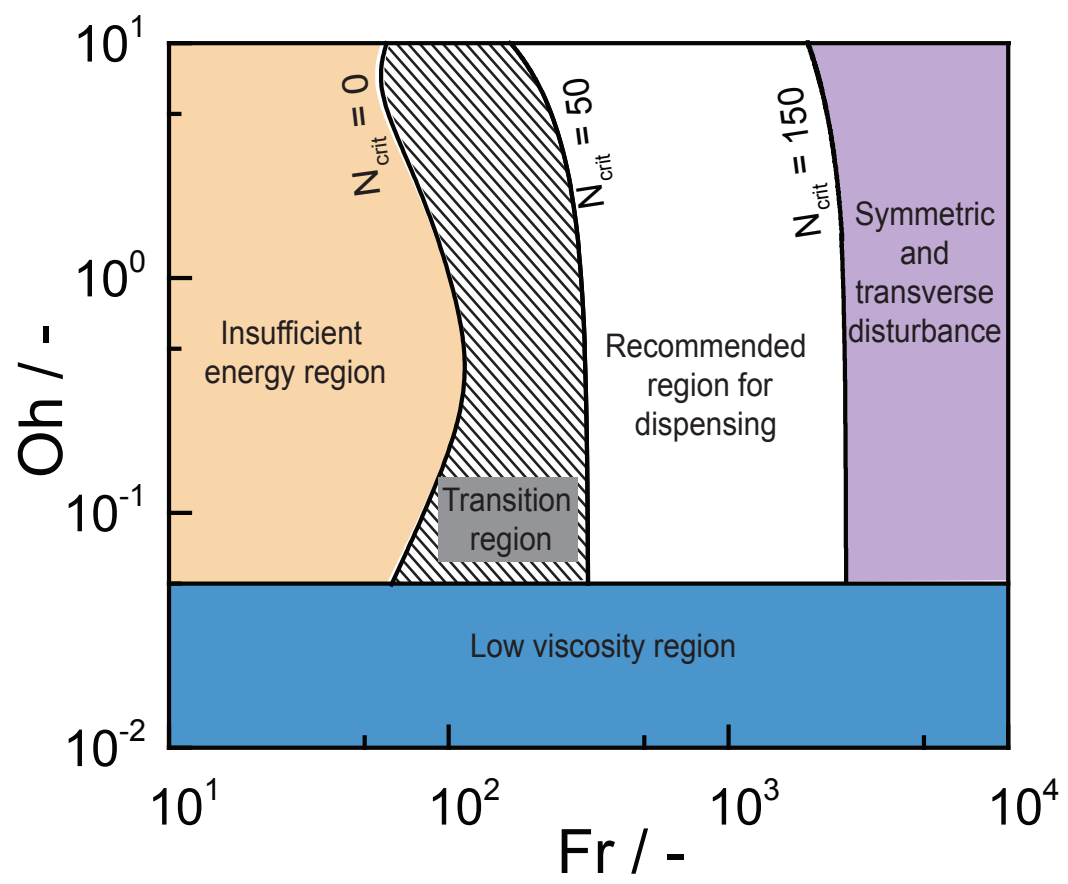

Figure 5: Map for dispensing liquids at various Froude and Ohnesorge numbers indicating preferred operation conditions.

our experience, we consider $N_{\text {crit }}$ values between 50 and 150 as suitable for micro dispensing. Of course, these values are not intended to be strict limits.

The far left region features relatively low $F r$ numbers. Here, the kinetic energy is not sufficient to establish a continuous jet and the liquids drip from the nozzle. Adjacent to the insufficient energy region, there is a regime that may be used for continuous dispensing of liquids. Here, stable jets are observed but $N_{\text {crit }}$ is below 50 which we consider as the lower limit for good dispensing; hence, we label it as the transition region. The region $50 \lesssim N_{\text {crit }} \lesssim 150$ is recommended for dispensing. Here, the break-up ratio of the jet, at a given $O h$ number, is almost linearly increasing with the 
Fr number. From the track of the isolines, we can conclude that a liquid with a low $O h$ number requires dispensing with a higher $\mathrm{Fr}$ number (kinetic energy) to achieve the same jet length as a very high $O h$ number liquid. On the right-hand side, there is another unfavorable region where increasing the Fr number (jet velocity) decreases the break-up ratio, cf. Figure 1a). In other words, the high Fr number triggers the development of symmetric and transverse disturbances which facilitates the instability of the jet interface. This region is named accordingly and is not recommended for dispensing.

\subsection{Jet Shape}

When a Newtonian liquid is dispensed under laminar conditions through a nozzle, the velocity profile is parabolic due to the no-slip condition at the liquid-solid interface. When the liquid exits the nozzle into a rather inviscid medium like air, the velocity profile changes, as a result of the changing boundary condition, which can alter the jet radius [59, 60]. For a vertical

jet, the jet shape is not only influenced by the liquid properties and the jet velocity but also by the acceleration due to gravity [59]. The NavierStokes equations can be employed but analytical solutions are only available for strong simplifications that reduce the complexity of the nonlinear partial differential equations. If we consider that there is a large density difference between jet and surrounding, and that there is only a very moderate change of the interface profile along the jet length, the nonlinear terms in the Navier-Stokes equation can be neglected. The respective analytical solution corresponds to eq. (2) with the coefficients $m=1$ and $n=4$; the derivation is given in the supplementary information. However, the omission of the nonlinear terms implies that this solution is only a good description for (rather) 
gravity-driven jets where inertial forces have no or little relevance. Indeed, comparison with the literature reveals that eq. (2) with $m=1$ and $n=4$ is used to describe the shape of jets with $0 \lesssim F r \lesssim 1$ and $0.03 \lesssim W e \lesssim 6.2$; cf. e.g. Refs. [45, 52, 60, 61].

In our experiments, we observe generally two different behaviors with respect to the continuous jet shape; i.e., contracting and non-contracting jets. Figure 6a) shows a jet that is dispensed at a $\mathrm{Fr}, \mathrm{We}$ and $\mathrm{Oh}$ number of 258, 12 and 1.98, respectively. In this case, we first observe a sudden contraction in the jet diameter in a short distance from the nozzle. However, the jet radius quickly recovers to approximately the nozzle diameter. This sudden contraction is related to the change of boundary conditions at the exit of the nozzle. The jet radius then continuously decreases over the jet length until the interface noticeably deforms. When the deformation becomes too strong, the jet breaks up and droplets are formed. The insets show magnifications of these different regimes: i) sudden contraction and recover of the jet radius followed by a continuous moderate contraction and ii) interface deformations indicating Rayleigh instability regime. A mathematical description of the continuous moderate contracting jet shape is desired to control the resolution of the printing process by the distance between nozzle and substrate. However, we also observe jets without a noticeable contraction in our experiments, cf. Figure 6b). These continuous jets have an approximately constant radius but still collapse after a certain length. This behavior simplifies the dispensing process since the resolution of the printing process is independent of the nozzle to substrate distance, provided there is no splashing induced by 
a)
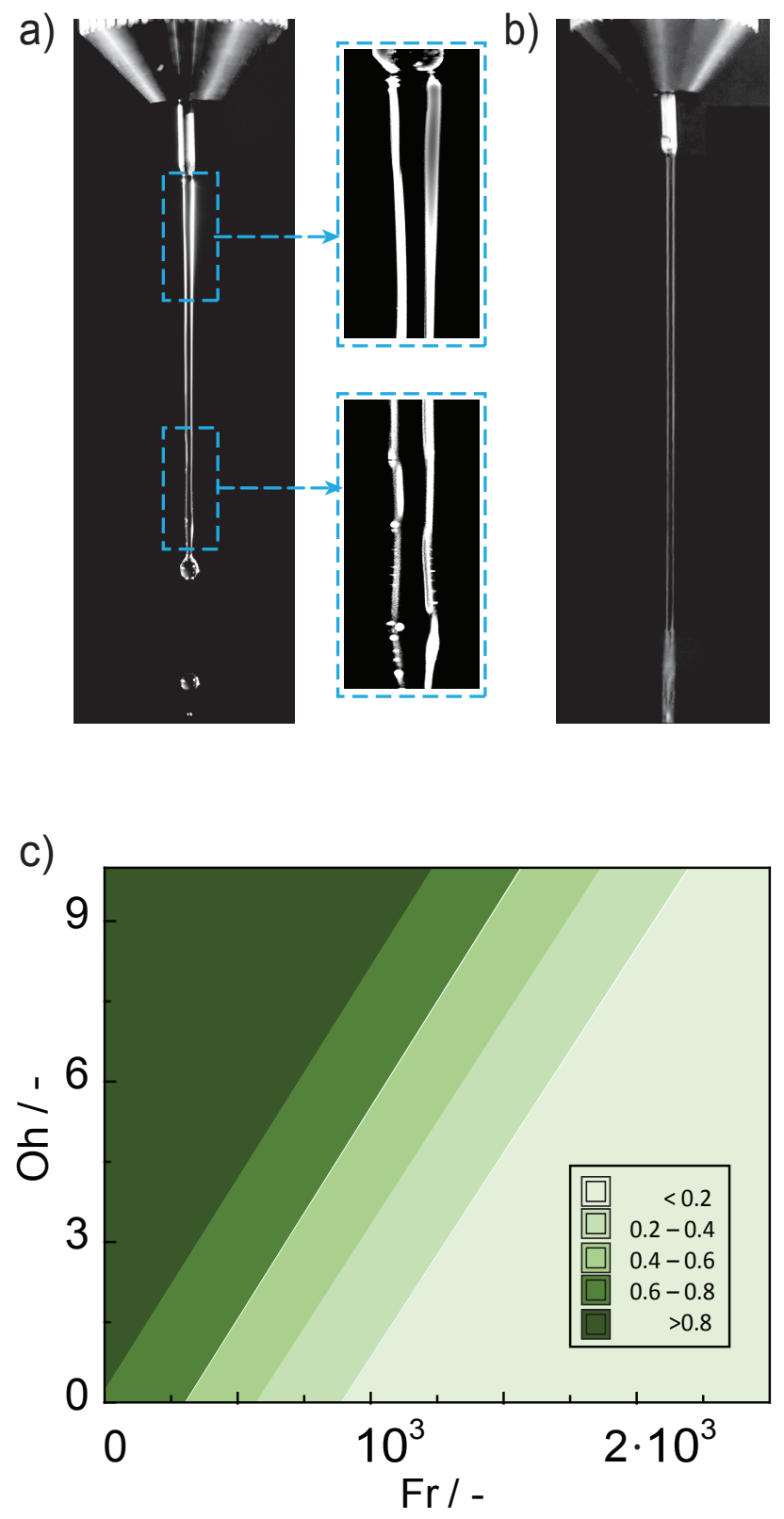

Figure 6: a) Shape of a contracting jet dispensed at $F r=258$ and $O h=1.98$. Insets show the magnification of the continuously contracting but stable jet and the Rayleigh instability region; b) Shape of a non-contracting jet at $F r=1937$ and $O h=0.05$; c) Contraction probability contour plot for jets with different $F r$ and $O h$ numbers. 
the high impact velocities. We observe this behavior at rather high Fr numbers. Here, it seems that the inertial force is entirely dominating, so that the gravitational force is not sufficient to elongate (contract) the jet. To obtain an insight of the conditions which result in non-contracting jet shapes, we use our experimental data and perform a binary logistic regression. The outcome of this data analysis is given in Figure 6c). This contour plot gives the probability whether a jet, at a given $\mathrm{Fr}$ and $\mathrm{Oh}$ number, measurably contracts before the Rayleigh instability regime. On a simplified level, we observe that a jet contraction is most likely for low $F r$ numbers. A large $O h$ number expands the Fr number range where contraction is (probably) observed. This is understood when we again use the interpretation $O h \sim F_{\text {vis }} / \sqrt{F_{\text {in }} F_{\text {cap }}}$. Here, we see that an increasing $O h$ number represents a decreasing influence of the inertia, which diminishes the shape-stabilizing influence of the high $\mathrm{Fr}$ number. Consequently, non-contracting continuous jets appear most likely for high $\mathrm{Fr}$ and rather low $\mathrm{Oh}$ numbers.

Application of eq. (2) with $m=1, n=4$, or any other constant values, to describe the contracting jet shape in our experiments is not feasible. This equation is only a good approximation for low $F r$ number jets. In the next section, we apply a heuristic correction to the jet shape eq. (2) to expand its applicability to inertia-driven jets with high $\mathrm{Fr}$ numbers.

\subsubsection{Modified Jet Shape Equation}

At first, we analyze the behavior of the inertia-driven but still contracting jet shape by selecting eight exemplary experiments which cover a wide range of parameters. In detail, we choose experiments with $\mathrm{Fr}$ and $\mathrm{Oh}$ numbers 
varying from around 40 to 1000 and 0.05 to 6 , respectively. Next, we perform a regression of the $\bar{r}_{j}$ and $\bar{x}$ data of the jet interface to eq. (2) with $m=1, n$ $=4$. However, the utilization of the actual $F r$ number in the regression does not have success. We therefore treat it as a fitting parameter which results in a very good regression quality. Table 2 gives the experimental parameters along with the fitted $\mathrm{Fr}$ number arising from the data regression.

Table 2: Actual and fitted non-dimensional groups for the regression of the jet shape eq. (2) $(m=1, n=4)$ to experimental data.

\begin{tabular}{|c|c|c|c|c|}
\hline Experiment & $F r$ & $W e$ & $O h$ & $F r_{f i t}$ \\
\hline Exp-1 & 43.98 & 1.63 & 5.00 & 1.63 \\
\hline Exp-2 & 119.58 & 5.60 & 1.98 & 2.10 \\
\hline Exp-3 & 131.37 & 1.90 & 6.32 & 2.0 \\
\hline Exp-4 & 241.73 & 15.41 & 0.20 & 2.53 \\
\hline Exp-5 & 335.77 & 21.40 & 0.20 & 3.54 \\
\hline Exp-6 & 596.92 & 38.00 & 0.20 & 4.83 \\
\hline Exp-7 & 718.48 & 43.91 & 0.05 & 6.20 \\
\hline Exp-8 & 1050.15 & 62.26 & 0.48 & 9.17 \\
\hline
\end{tabular}

We observe that the fitted $\mathrm{Fr}$ number is always considerably lower than the actual one. A plot of the fitted $F r$ number versus the $W e$ number reveals the linear correlation

$$
F r_{f i t} \approx 1+\frac{W e}{8} .
$$

Hence, our heuristic correction results in a modified jet shape equation for 
inertia-driven jets according to

$$
-\bar{x}=\left(1+\frac{W e}{8}\right)\left(\frac{1}{\bar{r}_{j}^{4}}+\frac{4}{\bar{r}_{j}} \frac{1}{W e}-\left(1+\frac{4}{W e}\right)\right) .
$$

This equation indicates that the shape of an inertia-driven jet mainly depends on the ratio of inertial to surface tension forces without the influence of the gravitational force. This is also indicated by contraction probability contour plot Figure 6c). In the next subsection, we validate eq. (11).

\subsubsection{Validation of the Modified Jet Shape Equation}

We test the quality of the modified equation by comparing the computed and measured jet shape as given in Figure 7a). Here, we use experiments from the regression data in Table 2. All shapes feature a considerable contraction over the jet length. The dimensionless jet radius decreases from one to roughly half of the initial radius. We observe that jets with low $F r$ numbers feature a relatively-higher contraction which as already shown qualitatively in the binary regression plot Figure 6b). Comparison of computed and experimental interface profiles show an excellent agreement given the simple methodology that we apply to correct the jet shape equation. Next, we validate the modified equation and test whether it can be used to predict

jet shapes from experiments that were not part of the regression data, cf. Figure 7b). Again, we find good agreement which confirms that the modified model can be used to predict the interface profile in a regime of $40 \lesssim F r \lesssim$ $1500 ; 1 \lesssim W e \lesssim 100$ and $0.05 \lesssim O h \lesssim 6$

\subsection{Dispensed Liquid Profile}

When a liquid is dispensed on a surface, the droplet (line) profile is far from equilibrium and the contact angle changes over time [62]. The transi- 


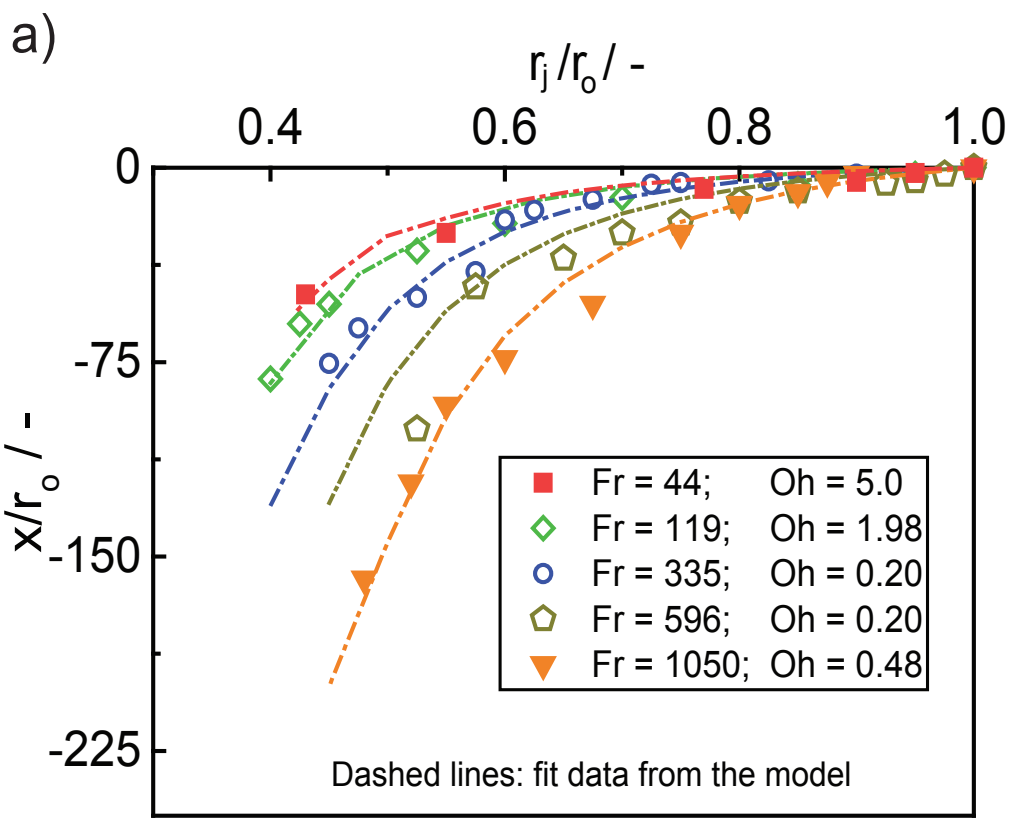

b)

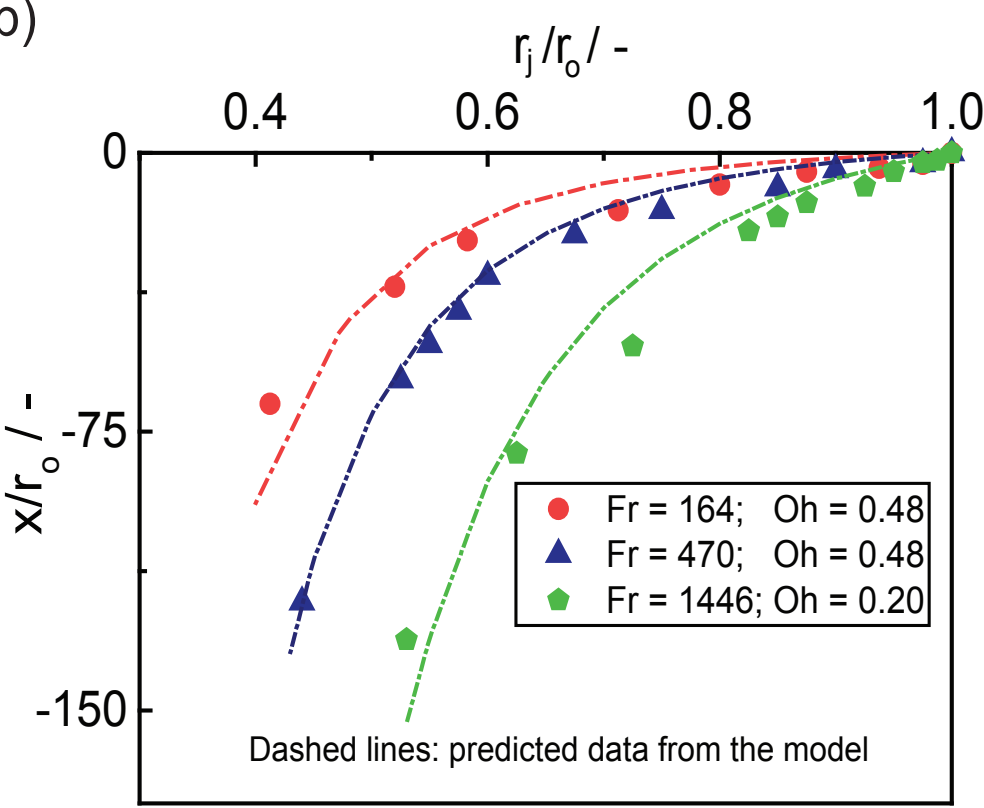

Figure 7: Comparison (validation) of measured and computed jet shapes for: a) Selected experiments from the regression data set in Table 2; and b) Experiments that are not part of the regression data set. 
tion to the equilibrium state is covered by dynamic models while the actual equilibrium is described with static models. Within the transition from dynamic to static state, the droplet goes through different phases; namely, kinematic, spreading, relaxation and wetting phases [62]. The maximum spreading radius of a dispensed droplet depends on the liquid density, viscosity, (initial) droplet diameter, solid-liquid interfacial tension along with the dispensing conditions (impact velocity). Several models are available in the literature which describe the dynamics of liquid spreading on a flat surface $[63,64,65,62,66]$.

The present work is not concerned with the spreading phase since a respective treatment would constitute a major research effort on its own. Here, we focus on the final equilibrium shape of a dispensed liquid on a flat surface - as illustrated in Figure 8a) - which is an important parameter in many industrial applications $[28,30,29]$. The equilibrium state is reached when: i) there is no further liquid movement on the substrate; ii) the interface between the air and liquid is symmetrical; iii) the liquid attained the substrate temperature; and iv) the volume of the liquid is conserved which means that there is no or hardly any evaporation [28].

Lubarda and Talke studied the static shape of a liquid droplet that is influenced by gravity. They used an ellipsoidal model based on the minimization of the Gibbs free energy and restricted the droplet shape to a spherical cap. This model accurately determines the droplet shape for contact angles below $120^{\circ}$ and droplet sizes on the order of the capillary length 
scale of the liquid [67]. Yeh et al. developed a model for 2D cylindrical and 3D axisymmetrical droplets based on the minimization of the Helmholtz free energy. This approach results in the augmented Young-Laplace equation for the droplet shape [68, 69]. Likewise, Berim and Ruckenstein [70] developed a model for cylindrical and axisymmetric droplet shape and the contact angle from a microscopic perspective using interaction potentials between the solid and liquid.

We use the Berim and Ruckenstein approach and correlate it to the dispensing parameters to obtain the 3D profile of a dispensed liquid and compare it with a corresponding experiment. In detail, we print a $2 \mathrm{~cm}$ line of a commercial carbon ink, with the liquid properties listed in Table 1, on a glass substrate. The ink is dispensed with a (lateral) print head velocity of $u=$ $4 \mathrm{~cm} / \mathrm{s}$ and with $\mathrm{Fr}, W e$ and $\mathrm{Oh}$ numbers of 295, 5.5 and 0.1, respectively. The spacing between nozzle and glass substrate is $5 \mathrm{~mm}$ and the diameter of the nozzle is $100 \mu \mathrm{m}$. Utilization of eq. (11) clarifies that for these conditions, the change in jet radius is negligible. The volume $V$ of the dispensed liquid is calculated according to

$$
V=\int_{0}^{t_{d}} v \cdot A d t
$$

where $A=\pi d^{2} / 4$ is the cross sectional area of the nozzle and $t_{d}$ is the dispensing time. The schematic of an equilibrium line profile on a flat surface is given in Figure 8a). The line has a length of $l$ and a width of $w$ and the cross-sectional profile of a cylindrical cap. The length of the dispensed line 


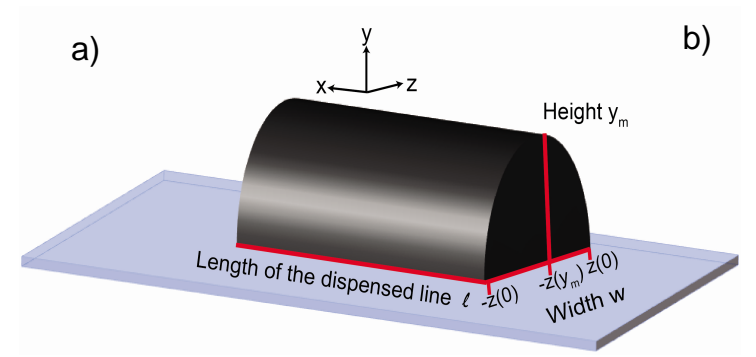

C)

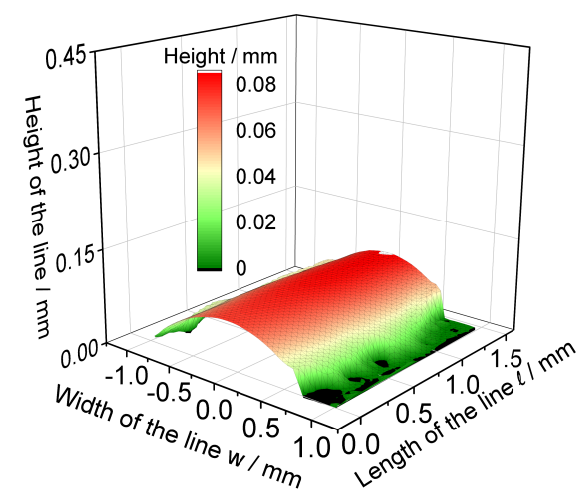

b)

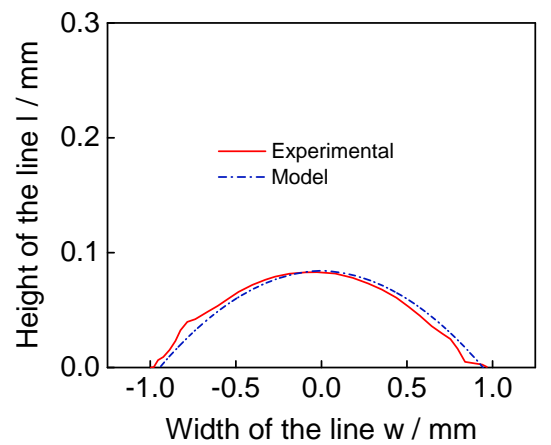

d)

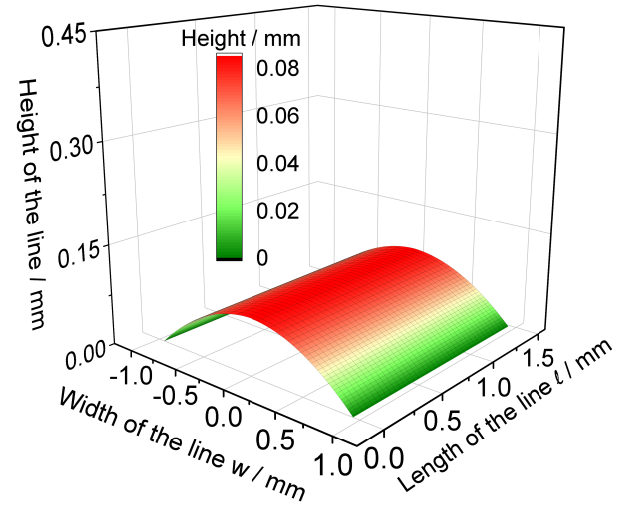

Figure 8: Dispensed liquid on a flat surface: a) Schematic 3D profile; b) Comparison of experimental and computed cross-sectional profile; c) Experimental 3D profile; and d) Computed 3D profile. 
is related to the dispensing time according to

$$
l=\int_{0}^{t_{d}} u d t
$$

A coordinate system for the cross-sectional profile is introduced where the width of the profile is a function of its height; that is, $z(y)$. The maximum height of the profile $y_{m}$ is located at the centerline at $z\left(y_{m}\right)$. The governing equation of the cross-sectional profile of a cylindrical droplet per unit length is then

$$
\frac{\frac{\mathrm{d}^{2} z}{\mathrm{~d} y^{2}}}{\left(1+\frac{\mathrm{d} z^{2}}{\mathrm{~d} y}\right)^{3 / 2}}+a \phi(y)-\lambda_{c}=0,
$$

where $\lambda_{c}$ is the Lagrange multiplier for the droplet volume. The correlation for the interaction energy $a$ and the function $\phi(y)$, which relates the size of the repulsive core $\kappa$ and the height of the profile, are defined as

$$
a=\frac{4}{3} \frac{\epsilon_{L S}}{\epsilon_{L L}} \frac{\rho_{s}}{\rho}\left(\frac{\kappa}{\eta}\right)^{4} ; \phi(y)=\frac{\kappa^{2}}{(\kappa+y)^{3}} .
$$

Here, $\epsilon_{i}$ are the strength of the interactions between liquid-liquid $(i=L L)$ or liquid-substrate $(i=L S) ; \rho_{s}$ is the number density of molecules in the substrate and $\eta$ is the radius of interaction. To convert eq. (14) into a first order differential equation, we substitute $\frac{\mathrm{d} z}{\mathrm{~d} y}=p(y)$ according to

$$
p(y)=\frac{\Psi(y)}{\sqrt{1-\Psi^{2}(y)}}
$$

where

$$
\Psi(y)=\lambda_{c} y+\frac{a \kappa^{2}}{2(\kappa+y)^{2}}+C .
$$


The cross-sectional (2D) profile of the dispensed line can then be obtained by solving

$$
z(y)=\int_{0}^{y} \frac{\Psi(y)}{\sqrt{1-\Psi^{2}(y)}} d y+\int_{0}^{y_{m}} \frac{\Psi(y)}{\sqrt{1-\Psi^{2}(y)}} d y
$$

where the second integral corresponds to the position of the contact line $z(0)$. Finally, the condition of a constant volume is engaged, where the dispensed liquid is correlated to the (3D) line profile according to

$$
V=\int_{0}^{t_{d}} v A d t=-2 \int_{0}^{t_{d}} u d t \int_{0}^{y_{m}} z(y) d y
$$

To compute the cross sectional profile with eq. (18), values for $a, \lambda_{c}, \kappa$, $y_{m}$ and $z(0)$ are needed. To calculate the maximum height $y_{m}$, eq. (19) with the appropriate dispensed volume constraint is solved. Generally, $a$ is a value less than 4 to keep the dispensed line stable [70]. Since we have no access to the properties required to estimate $a$ with eq. (15), it is used as a regression parameter. In detail, $a$ is chosen such that the experimental cross-sectional height and width of the profile are matched. The Lagrange multiplier $\lambda_{c}$ can then be calculated using

$$
\lambda_{c}=\frac{1}{y_{m}}\left[-2+\frac{a}{2}-\frac{a \kappa^{2}}{2\left(\kappa+y_{m}\right)^{2}}\right],
$$

where $\kappa=0.34 \mathrm{~nm}$ as the size of the repulsive core is used, cf. Ref.[71]. The numerical integrations to solve for the cross-sectional and the line profile are performed using the secant method. 
Figure $8 \mathrm{~b}$ ) allows for the comparison of the cross-sectional profiles obtained from model and experiment. A very good agreement is found when $\mathrm{n}$ interaction energy of $a=3.97$ is sued which fulfills the constrain $a<4$. Figure 8c) and d) contain a $1.5 \mathrm{~mm}$ segment of a measured and computed 3D profile, respectively. We find good agreement between the profiles both having a maximum height of $y_{m}=0.08 \mathrm{~mm}$ and a width $w \approx 2 \mathrm{~mm}$. Differences are obvious at the contact lines. These are mainly related to the evaporation of the ink and wetting/de-wetting effects which are not captured by the model. Nevertheless, the comparison proves that the present approach captures the most important features of the micro dispensing process.

\section{Concluding Remarks}

This study is concerned with various aspects of a direct-write micro dispensing process. In general, successful dispensing depends on the distance between nozzle and substrate, the jet diameter when contacting the substrate, and the profile of the dispensed liquid on the substrate. In this work, dispensing of a wide range of Newtonian liquids with varying properties is investigated.

First, the break-up lengths (ratio) of continuous jets are analyzed since this is an indication for the maximum possible distance between nozzle and substrate. Buckingham $\Pi$ theorem and a set of experiments are employed to infer a phenomenological correlation between dimensionless groups describing the break-up ratio of a jet with respect to liquid and dispensing parameters. 
This work clarifies that the experimentally-observed break-up ratios can be well described with the Froude and the Ohnesorge number. The presence of the gravitational force in the problem is rather counter-intuitive since the micro dispensing results in inertia-driven jets and one would assume that gravity has no influence. Furthermore, correlations for gravity-driven jets that are available in literature utilize the Weber number which is the ratio of inertial to surface tension forces. However, the surface tension forces are already included in the Ohnesorge number and an useful correlation, which describes the experimental results using Ohnesorge and Weber number, could not be identified, which validates the present approach to include the Froude number. Knowledge obtained from experiments and phenomenological correlation is used to construct a map which identifies parameters for successful direct-write micro dispensing of liquids.

Scheuerman's equation is modified to describe the radius of the jet interface as a function of jet length as well as liquid and dispensing properties. The original equation utilizes both Froude and Weber number and is successfully applied to gravity-driven jets, but is not applicable for jet shapes from micro dispensing. The proposed modification results in a very good description of the shape of inertia-driven jets and is solely a function of the Weber number. This is in agreement with the current observation that at higher Froude (and low Ohnesorge) numbers, the jet interface does not or hardly contracts. The modified equation can be used to estimate the resolution of the dispensed liquids on a substrate. 
Finally, Berim and Ruckenstein's microscopic model for the 2D shape of a liquid droplet on a flat surface is engaged. The model is extended and correlated to the dispensing parameters so that it describes the 3D equilibrium profile of a liquid dispensed on a flat and homogenous substrate. The comparison between model and experiments shows very good agreement but at the contact lines where there is noticeable influence of evaporation and wetting/dewetting phenomena.

In this work, rather simple Newtonian liquids are utilized but microdispensing processes also use non-Newtonian suspensions or dispersions with

high solid concentrations. Hence, it would be most interesting to investigate break-up length, jet shape and line profile of Non-Newtonian liquids in future work. Additionally, the influence of the impact and the lateral dispensing velocity deserves further investigations.

\section{Acknowledgements}

The authors gratefully acknowledge Lars Hagberg for taking photographs of the continuous jet and the financial support from the Natural Sciences and Engineering Research Council of Canada (NSERC) and an Ontario Early Researcher Award.

\section{References}

[1] B. Berman, 3-d printing: The new industrial revolution, Bus. Horiz. 55 (2) (2012) 155-162. 
[2] K. J. McHugh, T. D. Nguyen, A. R. Linehan, D. Yang, A. M. Behrens, S. Rose, Z. L. Tochka, S. Y. Tzeng, J. J. Norman, A. C. Anselmo, X. Xu, Fabrication of fillable microparticles and other complex 3d microstructures, Science 357 (6356) (2017) 1138-1142.

[3] Y. Chen, X. Li, Z. Bi, G. Li, X. He, X. Gao, Stamp-assisted printing of nanotextured electrodes for high-performance flexible planar microsupercapacitors, Chem. Eng. J. 353 (2018) 499-506.

[4] R. Xiang, X. Liang, P. Li, X. Di, W. Xiang, A thermally stable warm wled obtained by screen-printing a red phosphor layer on the luag: Ce3+ pig substrate, Chem. Eng. J. 306 (2016) 858-865.

[5] K. D. Wise, K. Najafi, Microfabrication techniques for integrated sensors and microsystems, Science 254 (5036) (1991) 1335-1342.

[6] J. Wang, B. Tian, V. B. Nascimento, L. Angnes, Performance of screenprinted carbon electrodes fabricated from different carbon inks, Electrochim. Acta 43 (23) (1998) 3459-3465.

[7] A. Hierlemann, O. Brand, C. Hagleitner, H. Baltes, Microfabrication techniques for chemical/biosensors, Proc. IEEE 91 (6) (2003) 839-863.

[8] W. Wu, Inorganic nanomaterials for printed electronics: a review, Nanoscale 9 (22) (2017) 7342-7372.

[9] X. Chen, G. Shoenau, W. Zhang, Modeling of time-pressure fluid dispensing processes, IEEE Trans. Electron. Packag. Manuf. 23 (4) (2000) 300-305. 
[10] X. Chen, J. Kai, Modeling of positive-displacement fluid dispensing processes, IEEE Trans. Electron. Packag. Manuf. 27 (3) (2004) 157-163.

[11] J. Tong, H.-X. Huang, M. Wu, Simultaneously facilitating dispersion and thermal reduction of graphene oxide to enhance thermal conductivity of poly (vinylidene fluoride)/graphene nanocomposites by water in continuous extrusion, Chem. Eng. J. 348 (2018) 693-703.

[12] M. Hashemi, X. Chen, Theoretical investigation into the performance of the rotary-screw fluid dispensing process, Trans. CSME/de la SCGM $32(3-4)(2008) 325-332$.

[13] D. Rose, Microdispensing technologies in drug discovery, Drug Discovery Today 4 (9) (1999) 411-419.

[14] Y. Zhang, C. Liu, D. Whalley, Direct-write techniques for maskless production of microelectronics: A review of current state-of-the-art technologies, in: Electronic Packaging Technology \& High Density Packaging, 2009. ICEPT-HDP'09. International Conference on, IEEE, 2009, pp. $497-503$.

[15] M. Vaezi, H. Seitz, S. Yang, A review on 3d micro-additive manufacturing technologies, Int. J. Adv. Manuf. Technol. 67 (5-8) (2013) 1721-1754.

[16] A. M'barki, L. Bocquet, A. Stevenson, Linking rheology and printability for dense and strong ceramics by direct ink writing, Sci Rep. 7 (1) (2017) 6017.

[17] S. Lee, J. H. Kim, M. Wajahat, H. Jeong, W. S. Chang, S. H. Cho, J. T. Kim, S. K. Seol, Three-dimensional printing of silver microarchitectures 
using newtonian nanoparticle inks, ACS Appl. Mater. Interfaces 9 (22) (2017) 18918-18924.

[18] S. Liu, Y. Li, S. Xing, L. Liu, G. Zou, P. Zhang, Structure inheritance in nanoparticle ink direct-writing processes and crack-free nano-copper interconnects printed by a single-run approach, Materials 12 (9) (2019) 1559 .

[19] S. Mortezapour, H. Eslami, E. Nedaaee Oskoee, Rheology and morphology of no-slip sheared polymer nanocomposite under creep condition, J. Chem. Phys. 143 (3) (2015) 034901.

[20] C. E. Corcione, M. Frigione, D. Acierno, Rheological characterization of uv-curable epoxy systems: Effects of o-boehmite nanofillers and a hyperbranched polymeric modifier, J. Appl. Polym. Sci. 112 (3) (2009) $1302-1310$.

[21] H. Sun, Y. Kim, Y. C. Kim, I. K. Park, J. Suhr, D. Byun, H. Choi, K. Kuk, O. H. Baek, Y. Jung, et al., Self-standing and shape-memorable uv-curing epoxy polymers for three-dimensional (3d) continuous-filament printing, J. Mater. Chem. C 6 (12) (2018) 29963003.

[22] V. S. Voet, T. Strating, G. H. Schnelting, P. Dijkstra, M. Tietema, J. Xu, A. J. Woortman, K. Loos, J. Jager, R. Folkersma, Biobased acrylate photocurable resin formulation for stereolithography 3d printing, ACS Omega 3 (2) (2018) 1403-1408. 
[23] B. Derby, Inkjet printing of functional and structural materials: fluid property requirements, feature stability, and resolution, Annu Rev Mater Res. 40 (2010) 395-414.

[24] R. Houben, Equipment for printing of high viscosity liquids and molten metals, Ph.D. thesis, University of Twente (9 2012). doi:10.3990/1.9789036534048.

[25] C. C. Chang, E. D. Boland, S. K. Williams, J. B. Hoying, Direct-write bioprinting three-dimensional biohybrid systems for future regenerative therapies, J Biomed Mater Res Part B: Appl Biomater 98 (1) (2011) 160-170.

[26] I. R. Damian, I. L. Omocea, D. Broboana, C. Balan, Impact of newtonian liquid jets on smooth and patterned solid walls, Energy Procedia 112 (2017) 186-193.

[27] M. Thrasher, S. Jung, Y. K. Pang, C.-P. Chuu, H. L. Swinney, Bouncing jet: A newtonian liquid rebounding off a free surface, Phys. Rev. E 76 (5) (2007) 056319.

[28] X. Chen, H. Ke, Effects of fluid properties on dispensing processes for electronics packaging, IEEE Trans. Electron. Packag. Manuf. 29 (2) (2006) 75-82.

[29] E. Carrilho, A. W. Martinez, G. M. Whitesides, Understanding wax printing: a simple micropatterning process for paper-based microfluidics, Anal. Chem. 81 (16) (2009) 7091-7095. 
[30] Y. Yokoyama, H. Fukumoto, K. Endo, Y. Fujii, T. Iwasaki, One picoliter ejection of solder droplets by an advanced molten solder ejection method, in: Micro Electro Mechanical Systems, 2007. MEMS. IEEE 20th International Conference on, IEEE, 2007, pp. 321-324.

[31] R. P. Grant, S. Middleman, Newtonian jet stability, AIChE J. 12 (4) (1966) 669-678.

[32] L. Rayleigh, On the instability of jets, Proceedings of the London mathematical society 1 (1) (1878) 4-13.

[33] L. Rayleigh, On the capillary phenomena of jets, Proc. R. Soc. London 29 (196-199) (1879) 71-97.

[34] C. Weber, Zum zerfall eines flüssigkeitsstrahles, J. Appl. Math. Mech. 11 (2) (1931) 136-154.

[35] A. Haenlein, Über den zerfall eines flüssigkeitsstrahles, Forschung im Ingenieurwesen 2 (4) (1931) 139-149.

[36] P. Schweitzer, Mechanism of disintegration of liquid jets, J. Appl. Phys. 8 (8) (1937) 513-521.

[37] M. McCarthy, N. Molloy, Review of stability of liquid jets and the influence of nozzle design, The Chemical Engineering Journal 7 (1) (1974) $1-20$.

[38] E. Ibrahim, S. Marshall, Instability of a liquid jet of parabolic velocity profile, Chem. Eng. J. 76 (1) (2000) 17-21. 
[39] M. Birouk, N. Lekic, Liquid jet breakup in quiescent atmosphere: A review, At. Sprays 19 (6).

[40] A. Haenlein, Über den zerfall eines flüssigkeitsstrahles, Forschung auf dem Gebiet des Ingenieurwesens A 2 (4) (1931) 139-149.

[41] M. Etzold, A. Deswal, L. Chen, F. Durst, Break-up length of liquid jets produced by short nozzles, Int. J. Multiphase Flow 99 (2018) 397-407.

[42] J. Eggers, E. Villermaux, Physics of liquid jets, Rep. Prog. Phys. 71 (3) (2008) 036601.

[43] J. Duda, J. Vrentas, Fluid mechanics of laminar liquid jets, Chem. Eng. Sci. 22 (6) (1967) 855-869.

[44] N. Clarke, Two-dimensional flow under gravity in a jet of viscous liquid, J. Fluid Mech. 31 (3) (1968) 481-500.

[45] J. N. Anno, The mechanics of liquid jets, Lexington, Mass., DC Heath and Co., 1977. 118 p.

[46] K. Adachi, K. Tagashira, Y. Banba, H. Tatsumi, H. Machida, N. Yoshioka, Steady laminar round jets of a viscous liquid falling vertically in the atmosphere, AIChE J. 36 (5) (1990) 738-745.

[47] U. S. Sauter, H. W. Buggisch, Stability of initially slow viscous jets driven by gravity, J. Fluid Mech. 533 (2005) 237-257.

[48] G. Kirchhoff, Vorlesungen über Mechanik... Vierte Auflage hrsg. von W. Wien. 1897, Vol. 1, BG Teubner, 1897. 
[49] R. Scheuermann, Über die gestalt und die aufösung des fallenden flüssigkeitsstrahles, Ann. Phys. 365 (19) (1919) 233-259.

[50] L. Scriven, R. Pigford, Fluid dynamics and diffusion calculations for laminar liquid jets, AIChE J. 5 (3) (1959) 397-402.

[51] T. Kurabayashi, Fine atomization of liquid by rotary nozzle hole: 3rd report, on thickness of liquid flow, J. Jpn. Soc. Mech. Eng. 25 (160) (1959) 1266-1273. doi:10.1299/kikai1938.25.1266.

[52] J. Mitrovic, A. Ricoeur, Fluid dynamics and condensation-heating of capillary liquid jets, Int. J. Heat Mass Transf. 38 (8) (1995) 1483-1494.

[53] P.-G. De Gennes, Wetting: statics and dynamics, Rev. Mod. Phys. 57 (3) (1985) 827.

[54] L. Leger, J. Joanny, Liquid spreading, Rep. Prog. Phys. 55 (4) (1992) 431.

[55] M. Brenner, A. Bertozzi, Spreading of droplets on a solid surface, Phys. Rev. Lett. 71 (4) (1993) 593.

[56] D. Bonn, J. Eggers, J. Indekeu, J. Meunier, E. Rolley, Wetting and spreading, Rev. Mod. Phys. 81 (2) (2009) 739.

[57] G. Lu, X.-D. Wang, Y.-Y. Duan, A critical review of dynamic wetting by complex fluids: from newtonian fluids to non-newtonian fluids and nanofluids, Adv. Colloid Interface Sci. 236 (2016) 43-62.

[58] E. Buckingham, On physically similar systems; illustrations of the use of dimensional equations, Phys. Rev. 4 (4) (1914) 345. 
[59] K. Adachi, Laminar jets of a plane liquid sheet falling vertically in the atmosphere, J. Non-Newtonian Fluid Mech. 24 (1) (1987) 11-30.

[60] T. Massalha, R. M. Digilov, The shape function of a free-falling laminar jet: making use of bernoulli's equation, Am. J. Phys 81 (10) (2013) 733-737.

[61] H. D. Haustein, R. S. Harnik, W. Rohlfs, A simple hydrodynamic model of a laminar free-surface jet in horizontal or vertical flight, Phys. Fluids 29 (8) (2017) 082105.

[62] D. Vadillo, A. Soucemarianadin, C. Delattre, D. Roux, Dynamic contact angle effects onto the maximum drop impact spreading on solid surfaces, Phys. Fluids 21 (12) (2009) 122002.

[63] P. Ehrhard, S. H. Davis, Non-isothermal spreading of liquid drops on horizontal plates, J. Fluid Mech. 229 (1991) 365-388.

[64] R. Rioboo, M. Marengo, C. Tropea, Time evolution of liquid drop impact onto solid, dry surfaces, Exp. Fluids 33 (1) (2002) 112-124.

[65] C. Ukiwe, D. Y. Kwok, On the maximum spreading diameter of impacting droplets on well-prepared solid surfaces, Langmuir 21 (2) (2005) 666-673.

[66] J. B. Lee, S. H. Lee, Dynamic wetting and spreading characteristics of a liquid droplet impinging on hydrophobic textured surfaces, Langmuir 27 (11) (2011) 6565-6573. 
[67] V. A. Lubarda, K. A. Talke, Analysis of the equilibrium droplet shape based on an ellipsoidal droplet model, Langmuir 27 (17) (2011) 1070510713.

[68] E. Yeh, J. Newman, C. Radke, Equilibrium configurations of liquid droplets on solid surfaces under the influence of thin-film forces: Part i. thermodynamics, Colloids Surf., A 156 (1-3) (1999) 137-144.

[69] E. Yeh, J. Newman, C. Radke, Equilibrium configurations of liquid droplets on solid surfaces under the influence of thin-film forces: Part ii. shape calculations, Colloids Surf., A 156 (1-3) (1999) 525-546.

[70] G. O. Berim, E. Ruckenstein, On the shape and stability of a drop on a solid surface, J. Phys. Chem. B 108 (50) (2004) 19330-19338.

[71] S. H. Lee, P. J. Rossky, A comparison of the structure and dynamics of liquid water at hydrophobic and hydrophilic surfaces-a molecular dynamics simulation study, J. Chem. Phys. 100 (4) (1994) 3334-3345. 